RESEARCH HIGHLIGHT

\title{
It's just a phase: NLRP6 phase separations drive signaling
}

\author{
Rebecca E. Tweedell (iD) ${ }^{1}$ and Thirumala-Devi Kanneganti (iD ${ }^{1 凶}$ \\ (c) CEMCS, CAS 2021 \\ Cell Research (2022) 32:113-114; https://doi.org/10.1038/s41422-021-00594-7
}

\begin{abstract}
NLRP6 is a key innate immune sensor whose functions have remained understudied. In a recent Cell paper, Shen et al. evaluate the mechanism of NLRP6 activation and find that double-stranded RNA interacts with NLRP6 and leads to liquid-liquid phase separation that is critical for NLRP6dependent innate immune signaling.
\end{abstract}

Innate immunity is the first line of defense against infection. Cytosolic innate immune sensors detect pathogens and activate inflammatory signaling and cell death to eliminate infected cells. Some cytosolic sensors do this by forming multiprotein complexes known as inflammasomes. Canonical inflammasomes generally consist of a sensor, the adapter protein ASC, and the effector molecule caspase-1. Complex formation activates caspase- 1 to cleave its downstream substrates pro-IL-1 $\beta$, pro-IL-18, and the pore-forming molecule gasdermin $D$ (GSDMD). Formation of GSDMD pores in the cell membrane facilitates the release of IL-1 $\beta$ and IL-18 and induces cell death, pyroptosis. Several sensors in the Nod-like receptor (NLR) family have been implicated in inflammasome formation. ${ }^{1}$ NLRP6 is one such sensor; it induces inflammasome-dependent IL-18 release in colitis models in mice ${ }^{2}$ and senses bacterial lipoteichoic acid (LTA) for non-canonical inflammasome activation through caspase-11, without cleavage of GSDMD. ${ }^{3}$ However, NLRP6 also has multifaceted functions physiologically. Beyond inflammasome activation, it negatively regulates inflammation during bacterial infections. ${ }^{4}$ It has also been implicated in the control of intestinal viral infections through DHX15-NLRP6 sensing of RNA and subsequent regulation of interferon (IFN) signaling. ${ }^{5}$ How NLRP6 can achieve these multifaceted roles and whether it induces canonical inflammasome activation and pyroptosis have remained unclear.

Some molecules, such as the innate immune sensor cGAS, control the activation of multiple signaling pathways by employing liquid-liquid phase separation (LLPS) to form a flexible signaling platform for the activation of downstream molecules. ${ }^{6}$ This allows for amplification and control of the signaling and facilitates dynamic protein-protein interactions. Recent work now suggests that NLRP6 also undergoes LLPS upon interaction with double-stranded RNA (dsRNA). ${ }^{7}$ Gel shift or microscale thermophoresis assays show that direct binding occurs between NLRP6 and dsRNA, as well as between NLRP6 and LTA, which is predominantly mediated through the NLRP6 NACHT-LRR region. Furthermore, highly dynamic phaseseparated droplets are visible by fluorescence microscopy when NLRP6 and dsRNA are combined, and DHX15 can co-localize to these same droplets. ASC and caspase- 1 also co-localize with the NLRP6-dsRNA droplets, although formation of these more complex structures causes the droplets to lose their dynamism. In live cells, within an hour of dsRNA introduction, NLRP6 and dsRNA form puncta that undergo rapid recovery in FRAP assays. ${ }^{7}$ The addition of
ASC increases the recovery time, further supporting that the presence of ASC changes the fundamental properties of these complexes. Together, these data suggest that NLRP6 undergoes LLPS in response to ligand sensing to create a foundation for the activation of multiple signaling pathways. However, upon ASC recruitment, the complex commits to inflammasome formation and forms more solidified filaments (Fig. 1).

Phase separation relies on the ability of a protein to make multivalent interactions, forming structures with both itself and other molecules. Intrinsically disordered regions (IDRs) can facilitate these interactions ${ }^{8}$ and are important for NLRP6-dsRNA puncta formation. ${ }^{7}$ A K350-354A mutant of NLRP6, where evolutionarily conserved lysines in one NLRP6 IDR were mutated to alanines, exhibited reduced LLPS without affecting NLRP6 binding to viral RNA, suggesting that self-oligomerization contributes to LLPS. Additionally, the K350-354A mutation impairs NLRP6 inflammasome activation and reduces cell death in immortalized bone marrow-derived macrophages, ${ }^{7}$ further suggesting that LLPS is an important driving force for NLRP6 complex formation and inflammasome activation.

Given the diverse roles shown previously for NLRP6 in infection and disease models, ${ }^{2-5}$ the implications of LLPS and inflammasome activation in host defense require careful consideration. NLRP6 is highly expressed in hepatocytes and intestinal epithelial cells, ${ }^{7}$ suggesting that it may have a role in innate immune responses in these cell types. Murine hepatitis virus (MHV), which infects hepatocytes, induces formation of NLRP6-dsRNA puncta in mouse liver sections. ${ }^{7}$ In addition, MHV-induced IL-18 release is decreased in mice that lack NLRP6, though not to the same extent as in mice lacking ASC. ${ }^{7}$ NLRP3 also contributes to inflammasome activation during MHV infection ${ }^{9}$ and is likely responsible for the residual IL-18 release in NIrp6 $6^{-/}$mice. Loss of DHX15 has no impact on inflammasome activation in the liver during MHV infection, suggesting that MHV-mediated NLRP6 inflammasome activation is DHX15-independent. In addition, viral replication is increased in $\mathrm{NIrp6}^{-/-}$mice but unchanged in Dxh15 $15^{-/-}$mice. In MHV-infected livers of mice carrying the NLRP6 K350-354A mutation, which was shown in vitro to disrupt LLPS and reduce inflammasome activation, NLRP6-dsRNA puncta are absent, cell death is less frequent, and IL-18 levels are reduced. Similarly, during infection with the intestinal pathogen rotavirus, inflammasome activation in the intestine is reduced in both $\mathrm{Nlrp6}^{-1-}$ and NIrp6 ${ }^{\mathrm{K} 350-354 \mathrm{~A}}$ mice compared with wild type. Beyond the impact on NLRP6 inflammasome activation, LLPS also likely critically contributes to the ability of NLRP6 to carry out its multifaceted innate immune functions, as N/rp6 $6^{\mathrm{K} 350-354 \mathrm{~A}}$ mice also have decreased expression of IFNs and IFN-stimulated genes (ISGs) during MHV infection. ${ }^{7}$

${ }^{1}$ Department of Immunology, St. Jude Children's Research Hospital, Memphis, TN, USA. ${ }^{凶}$ email: Thirumala-Devi.Kanneganti@StJude.org 


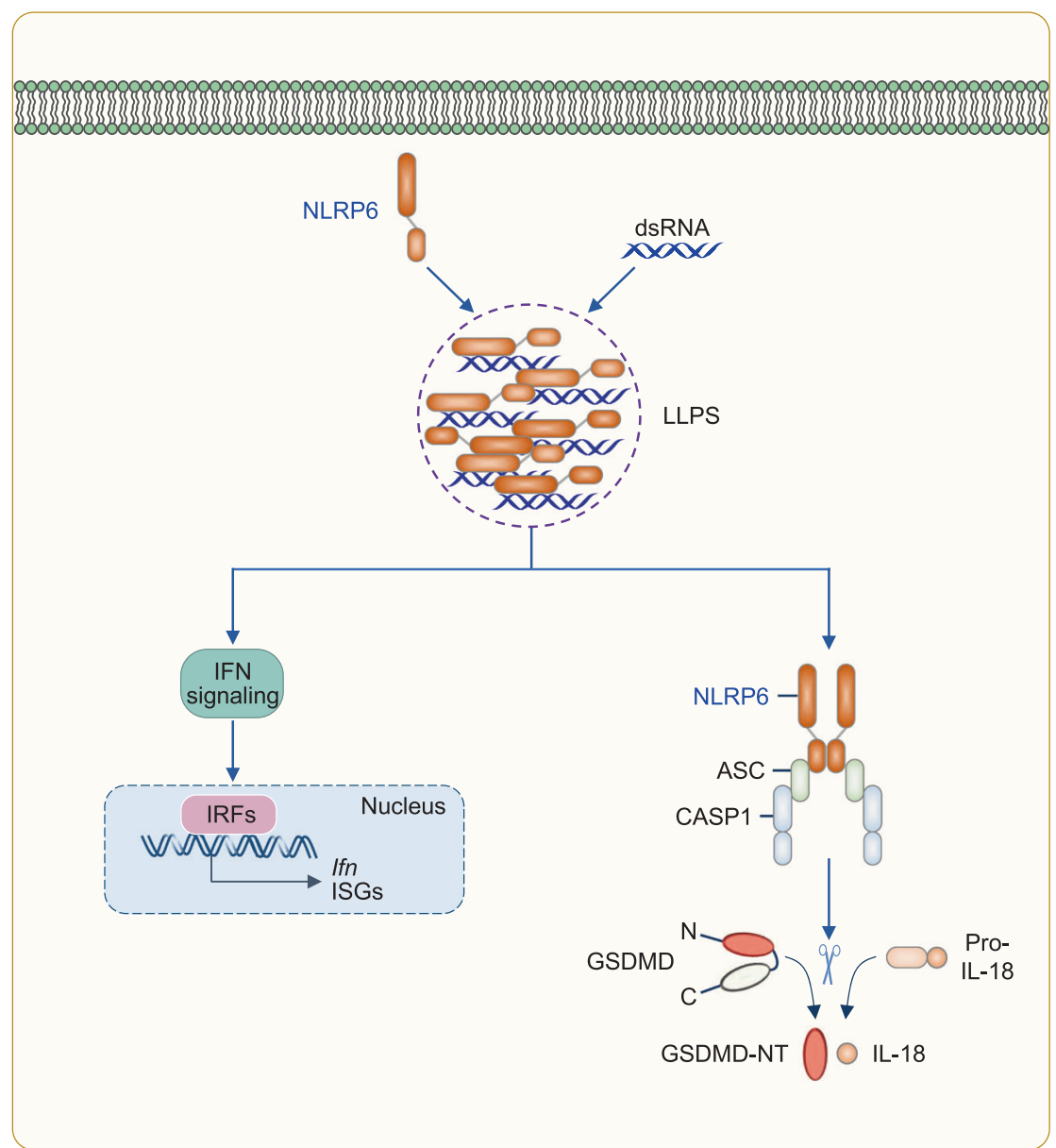

Fig. 1 LLPS facilitates NLRP6-mediated signaling. Shen et al. show that NLRP6 binds dsRNA and undergoes LLPS to form a membraneless compartment in the cytosol that can serve as a starting point for multiple signaling pathways. NLRP6 activates IFN signaling to induce expression of IFNs and ISGs. In addition, ASC can be recruited to the NLRP6 complex, leading to caspase-1 (CASP1) activation and cleavage of pro-IL-18 to its bioactive form and GSDMD to release its N-terminal region (GSDMD-NT). IRFs, interferon regulatory factors.

Overall, this new evidence suggests that the ability of NLRP6 to undergo LLPS contributes to its complex roles in innate immunity. The formation of a dynamic molecular platform provides flexibility in mediating multiple downstream signaling pathways and may help explain the previously disparate functions ascribed to NLRP6. In addition, given its influence across innate immune processes, NLRP6 could also be involved in other cell death processes beyond pyroptosis, such as PANoptosis. PANoptosis is defined as an inflammatory programmed cell death pathway regulated by the PANoptosome complex. PANoptosomes consist of caspase-8 and/or other caspases and cell death molecules, including inflammasome components, with functional redundancies. ${ }^{10}$ Given the LLPS induced by NLRP6 and ability of this phase transition to support dynamic protein-protein interactions and scaffolding activities, it is possible that LLPS could contribute to the formation of a PANoptosome or other types of higher order signaling scaffolds. Further studies will be needed to assess these possibilities. Improved understanding of NLRP6-mediated inflammasome and non-inflammasome signaling paves the way for identifying additional signaling molecules and defining new pathways that are critical for host defense.

\section{REFERENCES}

1. Christgen, S. \& Kanneganti, T. D. Curr. Opin. Immunol. 62, 39-44 (2020).

2. Elinav, E. et al. Cell 145, 745-757 (2011).

3. Hara, H. et al. Cell 175, 1651-1664 (2018).
4. Anand, P. K. et al. Nature 488, 389-393 (2012).

5. Wang, P. et al. Science 350, 826-830 (2015).

6. Du, M. \& Chen, Z. J. Science 361, 704-709 (2018).

7. Shen, C. et al. Cell 184, 5759-5774.e20 (2021).

8. Samir, P. \& Kanneganti, T. D. Trends Immunol. 40, 1082-1094 (2019).

9. Zheng, M. et al. J. Biol. Chem. 295, 26-38 (2020).

10. Lee, S. et al. Nature 597, 415-519 (2021).

\section{ACKNOWLEDGEMENTS}

Research in the Kanneganti lab is supported by grants from the US National Institutes of Health (Al101935, Al124346, Al160179, AR056296, and CA253095) and the American Lebanese Syrian Associated Charities to T.D.-K. The content is solely the responsibility of the authors and does not necessarily represent the official views of the National Institutes of Health.

\section{COMPETING INTERESTS}

The authors declare no competing interests.

\section{ADDITIONAL INFORMATION}

Correspondence and requests for materials should be addressed to Thirumala-Devi Kanneganti.

Reprints and permission information is available at http://www.nature.com/ reprints 